\title{
Research Article \\ Best Proximity Pairs Theorems for Continuous Set-Valued Maps
}

\author{
A. Amini-Harandi, ${ }^{1}$ A. P. Farajzadeh, ${ }^{2}$ D. O'Regan $^{\prime}{ }^{3}$ and R. P. Agarwal ${ }^{4}$ \\ ${ }^{1}$ Department of Mathematics, University of Shahrekord, Shahrekord 88186-34141, Iran \\ ${ }^{2}$ Department of Mathematics, Razi University, Kermanshah 67149, Iran \\ ${ }^{3}$ Department of Mathematics, National University of Ireland, Galway, Ireland \\ ${ }^{4}$ Department of Mathematical Sciences, Florida Institute of Technology, Melbourne, FL 32901, USA
}

Correspondence should be addressed to A. Amini-Harandi, aminih_a@yahoo.com

Received 15 July 2008; Accepted 16 September 2008

Recommended by Nan-jing Huang

A best proximity pair for a set-valued map $F: A \multimap B$ with respect to a set-valued map $G: A \multimap A$ is defined, and a new existence theorem of best proximity pairs for continuous set-valued maps is proved in nonexpansive retract metric spaces. As an application, we derive a coincidence point theorem.

Copyright (C) 2008 A. Amini-Harandi et al. This is an open access article distributed under the Creative Commons Attribution License, which permits unrestricted use, distribution, and reproduction in any medium, provided the original work is properly cited.

\section{Introduction and preliminaries}

Let $(M, d)$ be a metric space and let $A$ and $B$ be nonempty subsets of $M$. Let $d(A, B)=$ $\inf \{d(a, b): a \in A, b \in B\}$, and $\operatorname{Prox}(A, B)=\{(a, b) \in A \times B: d(a, b)=d(A, B)\} . A$ is said to be approximately compact if for each $y \in M$ and each sequence $\left(x_{n}\right)$ in $A$ satisfying the condition $d\left(x_{n}, y\right) \rightarrow d(y, A)$ there is a subsequence of $\left(x_{n}\right)$ converging to an element of $A$. Let

$$
\begin{aligned}
& B_{0}:=\{b \in B: d(a, b)=d(A, B) \text { for some } a \in A\}, \\
& A_{0}:=\{a \in A: d(a, b)=d(A, B) \text { for some } b \in B\} .
\end{aligned}
$$

Let $G: A \multimap A$ and $F: A \multimap B$ be set-valued maps. $\left(G\left(x_{0}\right), F\left(x_{0}\right)\right)$ is called a best proximity pair for $F$ with respect to $G$ if $d\left(G\left(x_{0}\right), F\left(x_{0}\right)\right)=d(A, B)$. Best proximity pair theorems analyze the conditions under which the problem of minimizing the real-valued function $x \rightarrow d(G(x), F(x))$ has a solution. In the setting of normed linear spaces, the best proximity pair problem has been studied by many authors; see [1-5]. In 2000, Sadiq Basha and Veeramani [4] proved the following theorem. 
Theorem 1.1. Let $E$ be a normed linear space. Let $A$ be a nonempty, approximately compact and convex subset of $E$ and let $B$ be a nonempty, closed and convex subset of $E$ such that $\operatorname{Prox}(A, B)$ is nonempty and $A_{0}$ is compact. Suppose that

(a) $F: A \multimap B$ is a set-valued map such that for every $x \in A_{0}, F(x) \cap B_{0} \neq \varnothing$, and for every $y \in B_{0}$, the fiber $F^{-1}(y)$ is open;

(b) for every open set $U$ in $A$, the set $\cap\{F(u): u \in U\}$ is convex;

(c) $g: A \rightarrow A$ is a continuous, proper, quasi-affine, and surjective single-valued map such that $g^{-1}\left(A_{0}\right) \subseteq A_{0}$.

Then there exists an element $x_{0} \in A_{0}$ such that

$$
d\left(g\left(x_{0}\right), F\left(x_{0}\right)\right)=d(A, B)
$$

In the rest of this section we recall some definitions and theorems which are used in the next section. Let $X$ and $Y$ be topological spaces with $A \subseteq X$ and $B \subseteq Y$. Let $F: X \multimap Y$ be a set-valued map with nonempty values. The image of $A$ under $F$ is the set $F(A)=\bigcup_{x \in A} F(x)$ and the inverse image of $B$ under $F$ is $F^{-}(B)=\{x \in X: F(x) \cap B \neq \varnothing\}$. Now $F$ is said to be

(a) closed if its graph, $\operatorname{Gr}(F)=\{(x, y) \in X \times Y: y \in F(x)\}$, is a closed set in product space $X \times Y$;

(b) upper semicontinuous, if for each closed set $B \subseteq Y, F^{-}(B)$ is closed in $X$;

(c) lower semicontinuous, if for each open set $B \subseteq Y$, the set $F^{-}(B)$ is open;

(d) continuous if $F$ is both lower semicontinuous and upper semicontinuous.

We say that $F: X \multimap Y$ is onto if $F(X)=Y$. If $F: X \multimap Y$ is onto then $F^{-}: Y \multimap X$, the lower inverse of $F$, is defined by $F^{-}(y)=\{x \in X: y \in F(x)\} . f: X \rightarrow Y$ is called a homeomorphism if $f$ is a bijective, continuous, and open map. We say that the set-valued mapping $F: X \multimap Y$ has a continuous selection if there exists a continuous function $f: X \rightarrow Y$ such that $f(x) \in$ $F(x)$ for each $x \in X$. We let

$$
\mathcal{S}(X, Y)=\{F: X \multimap Y: F \text { has a continuous selection }\}
$$

For a nonempty finite subset $D$ of $X$, let $\langle D\rangle$ denote the set of all nonempty finite subsets of $D$.

Definition 1.2. Let $X$ be a nonempty subset of a topological vector space $Y$. A set-valued map $F: X \multimap Y$ is said to be a generalized KKM mapping (GKKM) if for each nonempty finite set $\left\{x_{1}, \ldots, x_{n}\right\} \subseteq X$, there exist a set $\left\{y_{1}, \ldots, y_{n}\right\}$ of points of $Y$, not necessarily all different, such that for each subset $\left\{y_{i_{1}}, \ldots, y_{i_{k}}\right\}$ of $\left\{y_{1}, \ldots, y_{n}\right\}$, we have

$$
\operatorname{conv}\left\{y_{i_{1}}, \ldots, y_{i_{k}}\right\} \subseteq \bigcup_{j=1}^{k} F\left(x_{i_{j}}\right) .
$$

The following extension of the classical KKM principle in topological vector spaces is due to Chang and Zhang [6]. 
Theorem 1.3. Let $X$ be a nonempty subset of a topological vector space $Y$ and let $F: X \multimap Y$ be a GKKM mapping with closed values. Then, the family $\{F(x): x \in X\}$ has the finite intersection property, that is,

$$
\bigcap_{x \in A} F(x) \neq \varnothing \quad \text { for each } A \in\langle X\rangle
$$

Furthermore, if there exists an $x_{0} \in X$ such that $F\left(x_{0}\right)$ is a compact set in $Y$, then

$$
\bigcap_{x \in X} F(x) \neq \varnothing .
$$

Let $X$ be a nonempty subset of a topological vector space $Y$. Let $F: X \multimap Y$ and $G: Y \multimap Y$ be set-valued mappings such that for each nonempty finite set $\left\{x_{1}, \ldots, x_{n}\right\} \subseteq X$, there exists a set $\left\{y_{1}, \ldots, y_{n}\right\}$ of points of $Y$, not necessarily all different, such that for each subset $\left\{y_{i_{1}}, \ldots, y_{i_{k}}\right\}$ of $\left\{y_{1}, \ldots, y_{n}\right\}$, we have

$$
G\left(\operatorname{conv}\left\{y_{i_{1}}, \ldots, y_{i_{k}}\right\}\right) \subseteq \bigcup_{j=1}^{k} F\left(x_{i_{j}}\right)
$$

Then $F$ is called a generalized KKM mapping with respect to $G$. If the set-valued mapping $G: Y \multimap Y$ satisfies the requirement that for any generalized KKM mapping $F: X \multimap Y$ with respect to $G$ the family $\{\overline{F(x)}: x \in X\}$ has the finite intersection property, then $G$ is said to be have the KKM property. We denote

$$
\operatorname{KKM}(Y)=\{G: Y \multimap Y: G \text { has the KKM property }\}
$$

By Theorem 1.3, the identity map $I_{Y}$ has the KKM property. It is well known, and easy to see, that the continuous functions have the KKM property. Thus if a set-valued mapping G has a continuous selection, then $G$ has trivially the KKM property.

Let $(M, d)$ be a metric space and let $B(x, r)=\{y \in M: d(x, y) \leq r\}$ denote the closed ball with center $x$ and radius $r$. Let

$$
\operatorname{co}(A)=\bigcap\{B \subseteq M: B \text { is a closed ball in } M \text { such that } A \subseteq B\} .
$$

If $A=\operatorname{co}(A)$, we say that $A$ is an admissible subset of $M$. Note that $\operatorname{co}(A)$ is admissible and the intersection of any family of admissible subsets of $M$ is admissible. The following definition of a hyperconvex metric space is due to Aronszajn and Panitchpakdi [7].

Definition 1.4. A metric space $(M, d)$ is said to be a hyperconvex metric space if for any collection of points $x_{\alpha}$ of $M$ and any collection $r_{\alpha}$ of nonnegative real numbers with $d\left(x_{\alpha}, x_{\beta}\right) \leq r_{\alpha}+r_{\beta}$, we have

$$
\bigcap_{\alpha} B\left(x_{\alpha}, r_{\alpha}\right) \neq \varnothing .
$$


The simplest examples of hyperconvex spaces are finite dimensional real Banach spaces and $l_{\infty}$ endowed with the maximum norm.

Now we introduce an important class of metric spaces.

Definition 1.5 (see [8]). A nonexpansive retract metric space (i.e., an $N R$-metric space) $(M, E, r)$ consists of a metric space $(M, d)$, a convex subset $(E, \rho)$ of a metrizable topological vector space $(V, \rho)$ in which every closed ball is convex such that $(M, d)$ can be isometrically embedded into $(E, \rho)$ and $r: E \rightarrow M$ is a nonexpansive retraction.

Let $A \subseteq M$. We say that $A$ is $r$-convex if, for each $D \in\langle A\rangle, r(\operatorname{conv}(D)) \subseteq A$ (note we identify $M$ with the isometric embedding image set in $E$ ).

Remark 1.6. Every closed ball in $(E, \rho)$ is convex if and only if

$$
\rho\left(\alpha x_{1}+\beta x_{2}, \alpha y_{1}+\beta y_{2}\right) \leq \max \left(\rho\left(x_{1}, y_{1}\right), \rho\left(x_{2}, y_{2}\right)\right),
$$

for each $x_{1}, x_{2}, y_{1}, y_{2} \in E, \alpha+\beta=1, \alpha, \beta \geq 0$.

Examples 1.7. (a) Let $(X,\|\cdot\|)$ be a normed linear space. Let $E=X, \rho(x, y)=\|x-y\|$, and $r=I$ the identity mapping. Then $(X,\|\cdot\|)$ is a nonexpansive retract metric space. In this case $A \subseteq X$ is $r$-convex if and only if $A$ is convex.

(b) Let $(M, d)$ be a hyperconvex metric space. It is well known that there exists an index set $I$ and a natural isometric embedding from $M$ into $l_{\infty}(I)$. Also there exists a nonexpansive retraction $r: l_{\infty}(I) \rightarrow M$. Thus every hyperconvex metric space is an $\mathcal{N R}$ metric space. In hyperconvex metric spaces, every admissible set is $r$-convex. To see this, let $A \subseteq M$ be admissible and $D \in\langle A\rangle$. Then $r(\operatorname{conv}(D)) \subseteq \operatorname{co}(D)$ [9]. Since $A$ is admissible, then $\operatorname{co}(D) \subseteq \operatorname{co}(A)=A$. Thus $r(\operatorname{conv}(D)) \subseteq A$, which implies that $A$ is $r$-convex.

(c) Let $(X, d)$ be a metrizable Hausdorff topological vector space in which every closed ball is convex. Let $E=X, \rho(x, y)=d(x, y)$, and $r=I$ be the identity mapping. Then $(X, d)$ is an $\mathcal{N R}$-metric space. In this case, $A \subseteq X$ is $r$-convex if and only if $A$ is convex.

\section{Main theorems}

This section is devoted to main results on best proximity pairs.

Theorem 2.1. Let $(M, E, r)$ be an $N \mathcal{R}$-metric space. Let $A \subseteq M$ be nonempty, compact, $r$-convex, and let $B$ be a nonempty subset of $M$. Let $G: A \multimap A$ be a continuous, onto set-valued map with compact values such that $G^{-} \in \mathcal{S}(A, A)$. Let $F: A \multimap B$ be a continuous set-valued map with $r$ convex, compact values. Assume that $F(x) \cap B_{0} \neq \varnothing$, for each $x \in A$. Then there exists $x_{0} \in A$ such that

$$
d\left(G\left(x_{0}\right), F\left(x_{0}\right)\right)=d(A, B)
$$

Proof. Define a set-valued map $H: A \multimap A$ by

$$
H(y)=\{x \in A: d(G(x), F(x)) \leq d(G(y), F(x))\} .
$$


Since $y \in H(y)$, then $H(y) \neq \varnothing$ for each $y \in A$. We show that for each $y \in A, H(y)$ is closed and therefore is a compact subset of $A$. Let $x_{n} \in H(y)$ and $x_{n} \rightarrow x$. Since $F$ and $G$ are compact-valued, then there exist $s \in G(y), t \in F(x), u_{n} \in G\left(x_{n}\right)$, and $v_{n} \in F\left(x_{n}\right)$ such that

$$
\begin{gathered}
d\left(G\left(x_{n}\right), F\left(x_{n}\right)\right)=d\left(u_{n}, v_{n}\right), \\
d(G(y), F(x))=d(s, t) .
\end{gathered}
$$

Now $F$ is lower semicontinuous so for each $n \in \mathbb{N}$, there exists $t_{n} \in F\left(x_{n}\right)$ such that $t_{n} \rightarrow t$. Since $F(A)$ and $G(A)$ are compact and $F$ and $G$ are closed, without loss of generality, we may assume that $u_{n} \rightarrow u, v_{n} \rightarrow v, u \in G(x)$ and $v \in F(x)$. Therefore since $x_{n} \in H(y)$, we have

$$
\begin{aligned}
d(G(x), F(x)) & \leq d(u, v) \\
& =\lim _{n} d\left(u_{n}, v_{n}\right) \\
& =\lim _{n} d\left(G\left(x_{n}\right), F\left(x_{n}\right)\right) \\
& \leq \limsup _{n} d\left(G(y), F\left(x_{n}\right)\right) \\
& \leq \lim _{n} d\left(s, t_{n}\right) \\
& =d(s, t)=d(G(y), F(x)),
\end{aligned}
$$

which shows that $x \in H(y)$. Now, we prove that

$$
H: A \subseteq E \multimap E
$$

is a generalized KKM mapping with respect to $G^{-} \circ r$. To show this, suppose that $x_{1}, \ldots, x_{n}$ are in $A$ and take any $y_{0}$ with $y_{0} \notin \bigcup_{i=1}^{n} H\left(x_{i}\right)$. Then we have

$$
d\left(G\left(y_{0}\right), F\left(y_{0}\right)\right)>d\left(G\left(x_{k}\right), F\left(y_{0}\right)\right), \quad \forall k=1, \ldots, n .
$$

Let

$$
S\left(y_{0}\right):=\left\{x \in A: \exists y \in G(x) \text { such that } d\left(G\left(y_{0}\right), F\left(y_{0}\right)\right)>d\left(y, F\left(y_{0}\right)\right)\right\}
$$

Clearly $x_{k} \in S\left(y_{0}\right)$ for $k=1, \ldots, n$. Let $g: A \rightarrow A$ be a selection of $G$ (not necessary continuous). We take $z_{k} \in F\left(y_{0}\right)$ such that

$$
d\left(G\left(y_{0}\right), F\left(y_{0}\right)\right)>d\left(g\left(x_{k}\right), z_{k}\right), \quad \text { for } 1 \leq k \leq n \text {. }
$$


Let $\lambda_{i} \geq 0$ and $\sum_{i=1}^{n} \lambda_{i}=1$. Now $r$ is nonexpansive and Remark 1.6 yields (note we identify $M$ with the isometric embedding image set in $E$ )

$$
\begin{aligned}
d\left(r\left(\sum_{i=1}^{n} \lambda_{i} g\left(x_{i}\right)\right), r\left(\sum_{i=1}^{n} \lambda_{i} z_{i}\right)\right) & \leq \rho\left(\sum_{i=1}^{n} \lambda_{i} g\left(x_{i}\right), \sum_{i=1}^{n} \lambda_{i} z_{i}\right) \\
& \leq \max _{1 \leq i \leq n} \rho\left(g\left(x_{i}\right), z_{i}\right) \\
& =\max _{1 \leq i \leq n} d\left(g\left(x_{i}\right), z_{i}\right) \\
& <d\left(G\left(y_{0}\right), F\left(y_{0}\right)\right) .
\end{aligned}
$$

Since $F\left(y_{0}\right)$ and $A$ are $r$-convex, then

$$
r\left(\sum_{i=1}^{n} \lambda_{i} z_{i}\right) \in F\left(y_{0}\right), \quad r\left(\sum_{i=1}^{n} \lambda_{i} g\left(x_{i}\right)\right) \in A .
$$

Thus

$$
d\left(r\left(\sum_{i=1}^{n} \lambda_{i} g\left(x_{i}\right)\right), F\left(y_{0}\right)\right)<d\left(G\left(y_{0}\right), F\left(y_{0}\right)\right) .
$$

Hence, we deduce that (note that $G$ is onto and see the definition of $S\left(y_{0}\right)$ with $y=$ $\left.r\left(\sum_{i=1}^{n} \lambda_{i} g\left(x_{i}\right)\right)\right)$

$$
G^{-}\left(r\left(\operatorname{conv}\left\{g\left(x_{1}\right), \ldots, g\left(x_{n}\right)\right\}\right)\right) \subseteq S\left(y_{0}\right) .
$$

As $y_{0} \notin S\left(y_{0}\right)$, we have $y_{0} \notin G^{-}\left(r\left(\operatorname{conv}\left\{g\left(x_{1}\right), \ldots, g\left(x_{n}\right)\right\}\right)\right)$. Consequently,

$$
G^{-} \circ r\left(\operatorname{conv}\left\{g\left(x_{1}\right), \ldots, g\left(x_{n}\right)\right\}\right) \subseteq \bigcup_{i=1}^{n} H\left(x_{i}\right)
$$

Since $x_{1}, \ldots, x_{n}$ are arbitrary elements of $A$, then we deduce that for each subset $\left\{i_{1}, \ldots, i_{k}\right\} \subseteq$ $\{1, \ldots, n\}$ we have

$$
G^{-} \circ r\left(\operatorname{conv}\left\{g\left(x_{i_{1}}\right), \ldots, g\left(x_{i_{k}}\right)\right\}\right) \subseteq \bigcup_{j=1}^{k} H\left(x_{i_{j}}\right)
$$

Now since $G^{-} \in \mathcal{S}(A, A)$ and $r$ is continuous, then $G^{-} \circ r \in \mathcal{S}(E, A)$ and so $G^{-} \circ r$ has the KKM property. Hence the family $\{H(x): x \in A\}$ has the finite intersection property. Now since $H(x)$ is compact for any $x \in A$, we have immediately that $\bigcap_{x \in A} H(x) \neq \varnothing$. Therefore, there exists an $x_{0} \in A$ such that

$$
x_{0} \in \bigcap_{x \in A} H(x)
$$


Then, it is clear that

$$
d\left(G\left(x_{0}\right), F\left(x_{0}\right)\right) \leq d\left(G(x), F\left(x_{0}\right)\right) \quad \forall x \in A .
$$

Since $x_{0} \in A$, then

$$
d\left(G\left(x_{0}\right), F\left(x_{0}\right)\right)=\inf _{x \in A} d\left(G(x), F\left(x_{0}\right)\right)
$$

Since $G: A \multimap A$ is onto, then for each $y \in A$ there exists $x \in A$ such that $y \in G(x)$. Thus

$$
d\left(A, F\left(x_{0}\right)\right) \leq d\left(G(x), F\left(x_{0}\right)\right) \leq d\left(y, F\left(x_{0}\right)\right) .
$$

Hence

$$
\inf _{x \in A} d\left(G(x), F\left(x_{0}\right)\right)=d\left(A, F\left(x_{0}\right)\right)
$$

Pick $b \in F\left(x_{0}\right) \cap B_{0} \neq \varnothing$. Then there exists $a \in A$ such that $d(a, b)=d(A, B)$. Thus

$$
d\left(A, F\left(x_{0}\right)\right) \leq d(A, b) \leq d(a, b)=d(A, B)
$$

By (2.17), (2.19), and (2.20), we get

$$
d\left(G\left(x_{0}\right), F\left(x_{0}\right)\right) \leq d(A, B) .
$$

On the other hand, trivially

$$
d\left(G\left(x_{0}\right), F\left(x_{0}\right)\right) \geq d(A, B)
$$

Thus by (2.21) and (2.22), we get

$$
d\left(G\left(x_{0}\right), F\left(x_{0}\right)\right)=d(A, B) .
$$

Remark 2.2. (a) Let $G: A \rightarrow A$ be a single-valued homeomorphism. Then $G$ obviously satisfies all conditions of Theorem 2.1.

(b) There are many conditions under which $G^{-}$has a continuous selection [10-13].

The following corollary is immediate.

Corollary 2.3. Let $X$ be a normed linear space. Let $A \subseteq X$ be a nonempty compact convex and let $B$ be a nonempty subset of $X$. Let $G: A \multimap A$ be a continuous, onto set-valued map with compact values such that $G^{-} \in S(A, A)$. Let $F: A \multimap B$ be a continuous set-valued map with convex, compact values. Assume that $F(x) \cap B_{0} \neq \varnothing$, for each $x \in A$. Then there exists $x_{0} \in A$ such that

$$
d\left(G\left(x_{0}\right), F\left(x_{0}\right)\right)=d(A, B) .
$$


Remark 2.4. A similar result to that of Corollary 2.3 holds in every topological vector space in which every closed ball is convex. corollary.

Since hyperconvex metric spaces are $\mathcal{N R}$-metric spaces, then we have the following

Corollary 2.5. Let $(M, d)$ be a hyperconvex metric space. Let $A \subseteq M$ be a nonempty compact admissible and let $B$ be a nonempty subset of $M$. Let $G: A \multimap A$ be a continuous, onto set-valued map with compact values such that $G^{-} \in \mathcal{S}(A, A)$. Let $F: A \multimap B$ be a continuous set-valued map with admissible, compact values. Assume that $F(x) \cap B_{0} \neq \varnothing$, for each $x \in A$. Then there exists $x_{0} \in A$ such that

$$
d\left(G\left(x_{0}\right), F\left(x_{0}\right)\right)=d(A, B)
$$

Corollary 2.6. Let $(M, d)$ be a hyperconvex metric space. Let $A$ be a nonempty compact admissible subset of $M$. Let $G: A \multimap A$ be a continuous, onto set-valued map with compact values such that $G^{-} \in \mathcal{S}(A, A)$. Let $F: A \multimap M$ be a continuous set-valued map with admissible, compact values. Assume that $F(x) \cap A \neq \varnothing$, for each $x \in A$. Then there exists $x_{0} \in A$ such that

$$
G\left(x_{0}\right) \cap F\left(x_{0}\right) \neq \varnothing .
$$

Proof. Let $B=M$ and apply Corollary 2.5 (note $B_{0}=A$ ).

Remark 2.7. If we take $G=I_{A}$, Corollary 2.6 reduces to Corollary 3.5 of Kirk and Shin [14].

\section{References}

[1] M. A. Al-Thagafi and N. Shahzad, "Best proximity pairs and equilibrium pairs for Kakutani multimaps," Nonlinear Analysis: Theory, Methods \& Applications. In press.

[2] W. K. Kim and K. H. Lee, "Corrigendum to "Existence of best proximity pairs and equilibrium pairs" [J. Math. Anal. Appl. 316 (2006) 433-446]," Journal of Mathematical Analysis and Applications, vol. 329, no. 2, pp. 1482-1483, 2007.

[3] W. K. Kim and K. H. Lee, "Existence of best proximity pairs and equilibrium pairs," Journal of Mathematical Analysis and Applications, vol. 316, no. 2, pp. 433-446, 2006.

[4] S. Sadiq Basha and P. Veeramani, "Best proximity pair theorems for multifunctions with open fibres," Journal of Approximation Theory, vol. 103, no. 1, pp. 119-129, 2000.

[5] P. S. Srinivasan and P. Veeramani, "On best proximity pair theorems and fixed-point theorems," Abstract and Applied Analysis, vol. 2003, no. 1, pp. 33-47, 2003.

[6] S.-S. Chang and Y. Zhang, "Generalized KKM theorem and variational inequalities," Journal of Mathematical Analysis and Applications, vol. 159, no. 1, pp. 208-223, 1991.

[7] N. Aronszajn and P. Panitchpakdi, "Extension of uniformly continuous transformations and hyperconvex metric spaces," Pacific Journal of Mathematics, vol. 6, no. 3, pp. 405-439, 1956.

[8] A. Amini, M. Fakhar, and J. Zafarani, "KKM mappings in metric spaces," Nonlinear Analysis: Theory, Methods E Applications, vol. 60, no. 6, pp. 1045-1052, 2005.

[9] W. A. Kirk, B. Sims, and G. X.-Z. Yuan, "The Knaster-Kuratowski and Mazurkiewicz theory in hyperconvex metric spaces and some of its applications," Nonlinear Analysis: Theory, Methods $\mathcal{E}$ Applications, vol. 39, no. 5, pp. 611-627, 2000.

[10] H. Ben-El-Mechaiekh and M. Oudadess, "Some selection theorems without convexity," Journal of Mathematical Analysis and Applications, vol. 195, no. 2, pp. 614-618, 1995.

[11] J.-C. Hou, "Michael's selection theorem under an assumption weaker than lower semicontinuous in H-spaces," Journal of Mathematical Analysis and Applications, vol. 259, no. 2, pp. 501-508, 2001. 
[12] J. T. Markin, "A selection theorem for quasi-lower semicontinuous mappings in hyperconvex spaces," Journal of Mathematical Analysis and Applications, vol. 321, no. 2, pp. 862-866, 2006.

[13] D. Repovš and P. V. Semenov, Continuous Selections of Multivalued Mappings, vol. 455 of Mathematics and Its Applications, Kluwer Academic Publishers, Dordrecht, The Netherlands, 1998.

[14] W. A. Kirk and S. S. Shin, "Fixed point theorems in hyperconvex spaces," Houston Journal of Mathematics, vol. 23, no. 1, pp. 175-188, 1997. 